\title{
Characterization of non-tuberculous mycobacterial pulmonary disease in Nanjing district of China
}

\author{
Chunmei Hu', Lili Huang ${ }^{1}$, Min Cai ${ }^{2}$, Weixiao Wang ${ }^{3}$, Xudong Shi ${ }^{4}$ and Wei Chen ${ }^{3^{*}} \mathbb{D}$
}

\begin{abstract}
Background: Environmental bacteria, nontuberculous mycobacteria (NTM), are recognized as one of the major human infection pathogens. NTM are prone to be mistaken as multidrug-resistant Mycobacterium tuberculosis and challenge our fight against TB. In addition, treatment of NTM per se is intractable. Remarkably, the distribution of NTM pathogenic species is geographically specific. Thus, it is very important to summarize the prevalent features and clinical symptoms of NTM pulmonary disease. However, In Nanjing district, southeast China, there is no such a report.

Methods: Through investigating electronic medical records and analyzing data of clinical examination system (Lis), we retrospectively summarized the NTM species from 6012 clinical isolates from May 2017 to August 2018, and analyzed the association between NTM species and clinical symptoms.

Results: Of 6012 clinical specimens, 1461 (24.3\%) could grow in the MGIT 960 broth. Among these positive isolates, 1213 (83\%) were M. tuberculosis, 22 (1.5\%) were M. bovis, and 226 (15.5\%) were NTM. After deducting redundancy, those NTM specimens were confirmed from 154 patients, among which, 87 (56.5\%) patients met the full ATS/IDSA NTM disease criteria. The most common etiologic agent was M. intracellulare (70.1\%). NTM infection was associated with age, based on which $68.6 \%$ male patients and $77.8 \%$ female patients were over 50 years old. The older patients were more likely to have hemoptysis, but the younger patients were more likely to manifest chest congestion. Male patients were more likely to have shortness of breath and females were more likely to have hemoptysis. The most common radiographic presentation of NTM pulmonary disease was bronchiectasis, accounting for $39.1 \%$. Remarkably, multiple and thin-walled cavities were outstanding. The most frequent comorbidity of NTM disease was previous tuberculosis (64\%), followed by clinical bronchiectasis (19.5\%), HIV (19.5\%), and 6.9\% chronic obstructive pulmonary disease (COPD). There was no association between NTM species and clinical symptoms.

Conclusion: This study retrospectively investigated the prevalence of NTM pulmonary disease in Nanjing district, southeast China. Similar to Beijing area, north China, M. intracellulare was the major pathogenic NTM species. Clinical symptoms of the disease were not species-specific. Previous TB and HIV infection immensely enhanced risk of NTM disease.
\end{abstract}

Keywords: Nontuberculous mycobacteria, Prevalence, Pulmonary disease, Clinical symptom, Comorbidity

\footnotetext{
* Correspondence: weichennannan2017@163.com

${ }^{3}$ Clinical Research Center, the second hospital of Nanjing, Nanjing University

of Chinese Medicine, Zhongfu Road 1, Gulou District, Nanjing 210003,

Jiangsu, China

Full list of author information is available at the end of the article
}

(c) The Author(s). 2019 Open Access This article is distributed under the terms of the Creative Commons Attribution 4.0 International License (http://creativecommons.org/licenses/by/4.0/), which permits unrestricted use, distribution, and reproduction in any medium, provided you give appropriate credit to the original author(s) and the source, provide a link to the Creative Commons license, and indicate if changes were made. The Creative Commons Public Domain Dedication waiver (http://creativecommons.org/publicdomain/zero/1.0/) applies to the data made available in this article, unless otherwise stated. 


\section{Background}

Nontuberculous mycobacteria (NTM) are a large group of naturally-occurring mycobacteria, ubiquitous in the environment like soil and water [1-3]. NTM are very diverse organisms, comprising over 170 species with different virulence features [4]. Some species are opportunistic pathogens for human beings, which could cause severe pulmonary and non-pulmonary diseases $[2,5,6]$. In the past, NTM infection did not draw much attention since it was not transmitted from person to person, and was often thought as lab contamination and neglected. However, in recent two decades, with more advanced diagnostic laboratory techniques and increased awareness among clinicians, global reports for incidence of NTM infection have dramatically increased [7-9]. NTM are already recognized as one of the major emerging pathogens [10]. Like MTB, NTM usually cause chronic lung infection. Indeed, it is difficult to distinguish the clinical symptoms between NTM disease and TB, and NTM is often misdiagnosed as multidrug-resistant MTB [11]. Treatment of NTM infection is also challenging, requiring multiple antimycobacterial drugs, expert management and longer course [12]. Therefore, it is imperative to summarize the clinical characteristics of NTM pulmonary disease, to facilitate diagnosis and treatment of NTM infection in time.

The distribution of NTM pathogenic species is geographically specific $[13,14]$. M. avium complex is the major causative agent of NTM disease in Europe and North American [1]. In contrast, M. abscessus is most common in Asia and Oceania [13]. Even in Asia, the frequency of clinical isolation of NTM species is different between countries and cities. For example, $M$. simiae is the most frequently disease-causing NTM in Iran [15]. However, in South Korean, M. avium complex and $M$. abscessus are most commonly involved [16]. Thus, it's necessary to investigate local prevalence of NTM infection.

China is one of the TB high burden developing countries. According to the WHO report on global tuberculosis in 2018, new infection of TB in China was estimated as 889,000 in 2017, ranking second in the world [17]. Incidence of NTM infection in China also rapidly increased in recent decades $[18,19]$. So far, there are limited reports for characteristics of NTM pulmonary disease in China [20-23]. Especially, to our best knowledge, there is no evaluation of NTM prevalence in Nanjing, which is one of the most prosperous cities of Yangtze River Delta, southeast China. According to the report of Centers for Disease Control (CDC) of Nanjing in 2018, the annual incidence rate of TB is $26.43 / 100,000(2203 / 8,335,000)$ in Nanjing. The second hospital of Nanjing is the unique designated hospital for diagnosis and treatment of infectious diseases in Nanjing district. In this retrospective study, we summarized the demographic and clinical features of patients with NTM lung disease in our hospital and analyzed the association between clinical symptoms and different NTM species.

\section{Methods}

The retrospective study investigated and evaluated the patients with NTM pulmonary disease in Nanjing district of China from May 2017 to August 2018. A total of 6012 clinical specimens including sputum, endotracheal suction fluid, or alveolar lavage were processed following the previous protocol with sodium hydroxide-N-acetyll-cysteine and $0.7 \%$ chlorhexidine [24]. The pellets were cultured in the automated BACTEC MGIT 960 Mycobacteria culture system (BD), which usually reports positive results from two to four weeks and reports negative results after 42 days. According to the rules for Chinese clinical laboratory examination of tuberculosis [25], the positive mycobacterial growth was further cultured on two screening L-J medium, each containing either hydrazine thiophene-2-carboxylate $(\mathrm{TCH})$ or p-nitrobenzoic acid (PNB). The strain that did not grow on both mediums was recorded as $M$. bovis. MTB could grow only on the former, and NTM could grow on both. All those mediums were purchased from Zhuhai Beisuo biotech company.

All the NTM species were identified using Mycobacterium Species Identification kit (PCR-reverse dot blot) of Da An Gene company [26, 27]. With this kit, 22 NTM species are routinely identified in clinical molecular detection laboratory. Briefly, species-specific fragments are amplified by PCR with primers labelled by biotin at $5^{\prime}$ end. Fragments are harvested and hybridized with the nylon membrane in the stringent hybrid condition, on which species-specific probes are immobilized. The coloration of different loci corresponds to different NTM species. With this commercial diagnostic kit, 22 NTM species could be identified, including: $M$. smegmatis, $M$. intracellulare, $M$. kansasii, $M$. terrae, $M$. nonchromogenicum, $M$. tuberculosis complex, $M$. avium, M. scrofulaceum, M. xenopi, M. chelonae, M. gilvum, M. chelonae ss. Abscessus, M. phlei, M. fortuitum, M. porcinum, M. gordonae, M. triviale, M. gastri, M. vaccae, $M$. marinum, M. ulcerans, $M$. szulgai, $M$. diernhoferi, $M$. simiae. It is important to note that the kit could not differentiate $M$. chelonae ss. abscessus from $M$. chelonae, $M$. marinum from $M$. ulcerans, $M$. fortuitum from $M$. porcinum. For convenience's sake, we used $M$. abscessus to represent the complex of $M$. chelonae ss. abscessus and $M$. chelonae.

We collected and reviewed the electronic medical records of patients with NTM infection from medical system of our hospital. Evaluation of NTM lung disease was strictly based on the 2007 American Thoracic Society (ATS) and Infectious Disease Society of America (IDSA) guidelines [28], which briefly include the following: (1) 
pulmonary symptoms; (2) radiograph showing nodular or cavitary opacities; (3) at least one bronchial wash or lavage specimen positive, or repeated (two or more) sputum specimens positive. For each NTM patient, we analyzed their medical history, clinical symptoms, comorbidities, lab examination results and radiographic features. All data were analyzed by Chi-square test, Correction chi-square test, Fisher $P$ values, unifactor and multifactor logistics stepwise regression analysis. $p<0.05$ means statistical significance.

\section{Results}

A total of 6012 clinical specimens were cultured in the BACTEC MGIT 960 mycobacteria culture system and 1461 (24.3\%) showed growth. Among these positive isolates, 1213 (83\%) were MTB, 22 (1.5\%) were M. bovis, and $226(15.5 \%)$ were NTM. After deducting redundancy, those NTM specimens were confirmed from 154 patients. Considering culture results, radiographic features, medical records and clinical symptoms, 87 (56.5\%) patients met the full ATS/IDSA NTM disease criteria and were diagnosed with NTM lung disease. The remaining 67 NTM cases $(43.5 \%)$ did not meet all the criteria and were diagnosed as nonpathogenic colonization. The clinical records of 87 patients with NTM lung disease were available for review. Five different species of NTM were observed (Fig. 1). M. intracellulare was etiologic agent in most cases $(n=61,70.1 \%)$. M. abscessus (representing the complex of $M$. chelonae ss. abscessus and M. chelonae, refer to Methods) and $M$. avium respectively accounted for $11.5 \%$ of isolates from NTM patients $(n=10)$. A small number of cases were due to $M$. kansasii, $(n=5,7.5 \%)$ and M. gordonae ( $\mathrm{n}=1,1.1 \%)$. Most pathogenic NTM in this study were slowly growing mycobacteria except for M. abscessus, which is a rapidly growing mycobacterium.
Meanwhile, analysis of the erythrocyte sedimentation rates (ESR) of all NTM patients showed that $83.9 \%(73 / 87)$ of NTM disease patients had higher ESR, indicating chronic inflammation in those patients.

\section{Demographic feature of patients with NTM pulmonary disease}

The median age was 60 years for all 87 patients. Among them, 51 (58.6\%) were male with median age 63 and 36 (41.4\%) were female with median age 59 (Fig. 2). For all patients, $68.6 \%(35 / 51)$ male and $77.8 \%(28 / 36)$ female were over 50 years old, indicating that NTM infection was associated with age (Fig. 2). All five NTM species were found in male patients. In contrast, only $M$. intracellulare and $M$. abscessus were observed in the female patients.

\section{Radiographic characteristic of patients with NTM pulmonary disease}

To determine whether different NTM species caused different pulmonary damage, we analyzed the radiographic characteristic of NTM patients. As shown in Table 1, the most common radiographic presentation of NTM pulmonary infection was bronchiectasis, accounting for $39.1 \%$ (34/87). Cavity and nodules were observed in more than one third patients. Remarkably, multiple and thinwalled cavities were outstanding. 18.3 and $8.0 \%$ NTM patients suffered from lymphadenovarix and pleural/pericardial effusion, respectively. After statistical analysis, there was no association between NTM species and radiographic characteristics.

\section{Clinical manifestation of NTM pulmonary disease}

Presentation of NTM pulmonary infection included cough and expectoration, hemoptysis, chest congestion,

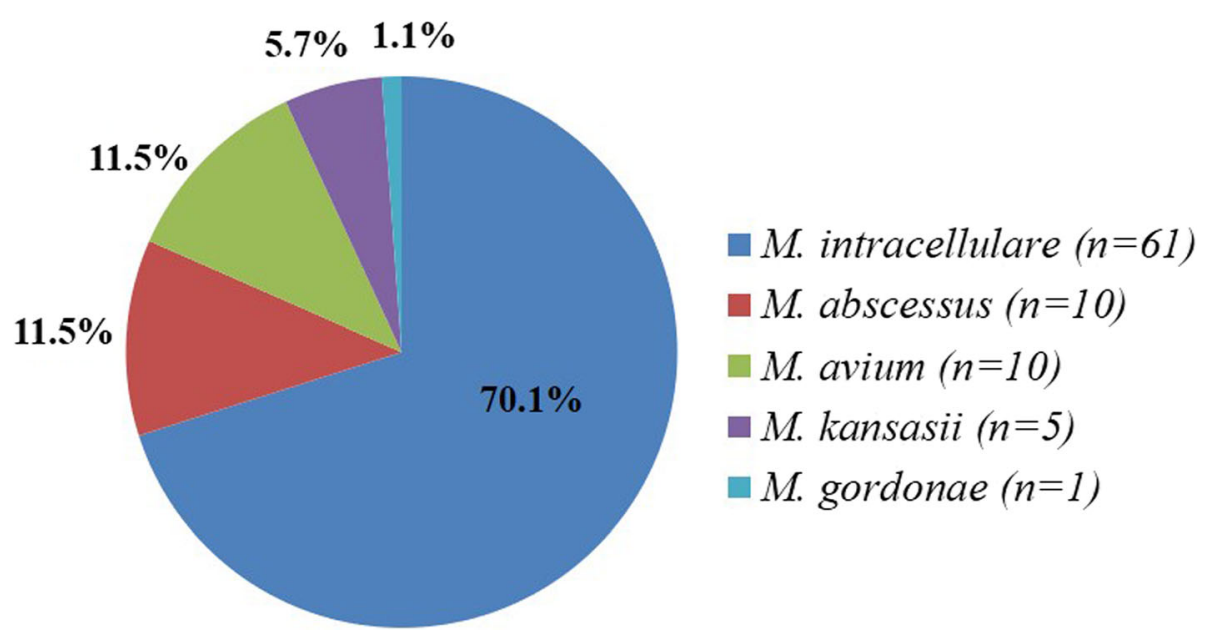

Fig. 1 Distribution of etiologic agents of NTM lung disease in Nanjing district of China from May 2017 to August 2018 ( $n=87)$. M. abscessus represented the complex of M. chelonae ss. abscessus and M. chelonae 


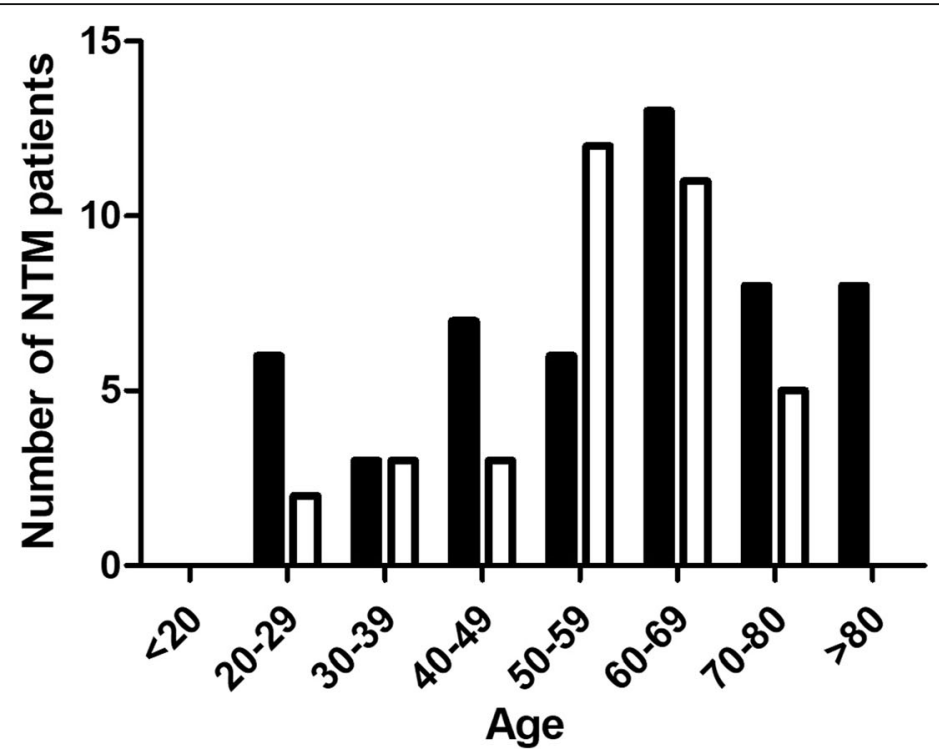

Male

$\square$ Female

shortness of breath, fever (as shown in Table 2). There was no association between clinical symptoms and NTM species. However, through Pearson chi square test, we did notice the association between shortness of breath and hemoptysis and gender. Male patients were more likely to have shortness of breath $\left(\chi^{2}=0.255 .41, p=\right.$ 0.020 ), and females were more likely to have hemoptysis $(\mathrm{x} 2=4.74, p=0.030)$. In addition, there was association between hemoptysis and chest congestion and age. The older patients were more likely to have hemoptysis $(r=$ $0.26, p=0.017$ ), but the younger patients were more likely to manifest chest congestion $(r=0.24, p=0.027)$.

\section{Comorbidities of NTM pulmonary disease}

Of 87 NTM patients, there were 30 cases (34.5\%) which had no associated complications. The most common comorbidity of NTM infection was previous TB (56/87) (Table 3), which showed that previous MTB infection significantly increased risk for further NTM infection. The second comorbidity was HIV infection. 17 NTM patients were HIV positive. Also, clinical bronchiectasis was associated with NTM infection (17/87). A small part of NTM patients meanwhile had COPD (6/87), hepatitis (5/87) and diabetes mellitus (4/87) (Table 3). Through multifactor regression analysis, comparing with female,

Table 1 Radiographic characteristics of different NTM species infection

\begin{tabular}{|c|c|c|c|c|c|c|c|}
\hline Presentations/No. of isolates & $\operatorname{MAB}(n=10)$ & $\operatorname{MAV}(n=10)$ & MKA $(n=5)$ & MIN $(n=61)$ & $\operatorname{MGO}(n=1)$ & Total87 & Percent \\
\hline Bronchiectasis & 7 & 2 & 4 & 20 & 1 & 34 & $39.1 \%$ \\
\hline Pulmonary Cavity & 6 & 2 & 2 & 23 & 0 & 33 & $37.9 \%$ \\
\hline Single cavity & 0 & 0 & 0 & 3 & 0 & 3 & $3.45 \%$ \\
\hline Multiple cavities & 6 & 2 & 2 & 20 & 0 & 30 & $34.48 \%$ \\
\hline Thick-walled cavity & 0 & 0 & 0 & 1 & 0 & 1 & $1.15 \%$ \\
\hline Thin-walled cavity & 6 & 2 & 2 & 22 & 0 & 32 & $36.78 \%$ \\
\hline Nodule & 5 & 4 & 2 & 22 & 0 & 33 & $37.9 \%$ \\
\hline Lymphadenovarix & 2 & 2 & 0 & 12 & 0 & 16 & $18.3 \%$ \\
\hline Pleural/Pericardial Effusion & 0 & 2 & 0 & 5 & 0 & 7 & $8.0 \%$ \\
\hline Destroyed lungs & 0 & 0 & 0 & 3 & 0 & 3 & $3.4 \%$ \\
\hline Pneumothorax & 0 & 0 & 1 & 1 & 0 & 2 & $2.3 \%$ \\
\hline Total Percent & 32 16.5\% & $168.2 \%$ & $136.7 \%$ & $13268 \%$ & $10.5 \%$ & 194 & \\
\hline
\end{tabular}


Table 2 Clinical symptoms of NTM lung disease

\begin{tabular}{|c|c|c|c|c|c|c|c|}
\hline Symptoms No. of isolates & $\operatorname{MAB}(n=10)$ & $\operatorname{MAV}(n=10)$ & $\operatorname{MKA}(n=5)$ & $\operatorname{MIN}(n=6)$ & $\mathrm{MGO}(n=1)$ & Total87 & Percent \\
\hline Cough/Expectoration & 8 & 9 & 5 & 45 & 1 & 68 & $78.2 \%$ \\
\hline Hemoptysis & 6 & 0 & 0 & 11 & 0 & 17 & $19.5 \%$ \\
\hline Chest Congestion & 1 & 0 & 0 & 7 & 0 & 8 & $9.2 \%$ \\
\hline Shortness of breath & 2 & 2 & 0 & 5 & 0 & 9 & $10.3 \%$ \\
\hline Fever & 1 & 5 & 3 & 15 & 0 & 24 & $27.6 \%$ \\
\hline Total & 18 & 16 & 8 & 83 & 1 & 126 & \\
\hline Percent & $14.3 \%$ & $12.7 \%$ & $6.3 \%$ & $65.9 \%$ & $0.8 \%$ & & \\
\hline
\end{tabular}

MAB M. chelonae ss. abscessus and M. chelonae complex, MAV M. avium, MKA M. kansasii, MIN M. intracellulare, MGO M. gordonae

male NTM patients were more likely to co-infect HIV $(\mathrm{OR}=20.92$, 95\% CI $(2.60,168.12))$ and COPD $(\mathrm{OR}=$ 10.46, 95\% CI $(1.23,88.97))$. Interestingly, among NTM species, $M$. intracellulare infection was more likely to combine with COPD $(\mathrm{OR}=0.18,95 \%$ CI $(0.04,0.97)$ ) and bronchiectasis $(\mathrm{OR}=0.18,95 \% \mathrm{CI}(0.04,0.72))$.

\section{Comparison analysis between patients of NTM disease and NTM colonization}

Besides 87 patients with NTM disease, there were still 67 cases of NTM colonization, which did not meet the full ATS/IDSA NTM disease criteria. To determine the risk factors for NTM disease, we performed comparison analysis between the two group patients. Through Chi-square test analysis, there was no significant difference between patients with NTM disease and NTM colonization regarding age, gender, radiographic characteristic and clinical presentation, except that NTM disease patients presented with more fever (27.6\%) (Table 4). Remarkably, of 87 NTM disease patients, 56 (64.4\%) patients had TB infection history and 17 patients were HIV-positive, which suggested that previous TB and HIV infection significantly increased the risk for NTM disease (Table 4).

\section{Discussion}

In our retrospective study, we investigated a total of 1461 culture-positive clinical specimens from respiratory patients. The isolation rate of NTM reached $15 \%$, but most of which did not cause disease. Among 87 NTM disease cases, five kinds of NTM species were detected, four slowly growing mycobacteria ( $M$. intracellulare, $M$. avium, $M$. kansasii, $M$. gordonae) and one rapidly growing mycobacterium ( $M$. abscessus). $M$. intracellulare was the predominant species, accounting for $70.1 \%$ of total disease-causing NTM, followed by $11.5 \% \mathrm{M}$. avium and $11.5 \%$ M. abscessus. These data were consistent with Zhang's report that $M$. intracellulare was the most common NTM species in China [23]. Published case reports and series showed that slender, older women were more susceptible to NTM lung infection, especially postmenopausal women [29]. In our study, we did find NTM infection was associated with age, not gender. Actually, with the increasing life span and more availability of people in geriatric age group, the increase in incidence of NTM lung disease is not surprising.

Cough and expectoration were the main clinical presentation of NTM chronic infection, followed by hemoptysis

Table 3 Comorbidities of NTM lung disease

\begin{tabular}{|c|c|c|c|c|c|c|c|}
\hline Comorbidities No. of isolates & $\operatorname{MAB}(n=10)$ & $\operatorname{MAV}(n=10)$ & MKA $(n=5)$ & $\operatorname{MIN}(n=6)$ & $\mathrm{MGO}(n=1)$ & Total87 & Percent \\
\hline Previous TB & 8 & 6 & 0 & 41 & 1 & 56 & $64.4 \%$ \\
\hline Clinical Bronchiectasis & 6 & 0 & 0 & 11 & 0 & 17 & $19.5 \%$ \\
\hline HIV positive & 0 & 8 & 4 & 5 & 0 & 17 & $19.5 \%$ \\
\hline COPD & 0 & 0 & 1 & 5 & 0 & 6 & $6.9 \%$ \\
\hline Hepatitis & 2 & 0 & 0 & 3 & 0 & 5 & $5.7 \%$ \\
\hline DM & 0 & 0 & 0 & 4 & 0 & 4 & $4.6 \%$ \\
\hline Hypertension & 1 & 0 & 0 & 3 & 0 & 4 & $4.6 \%$ \\
\hline Cancer & 0 & 0 & 0 & 3 & 0 & 3 & $3.4 \%$ \\
\hline AS & 0 & 1 & 0 & 0 & 0 & 1 & $1.1 \%$ \\
\hline Pulmonary fibrosis & 1 & 0 & 0 & 3 & 0 & 4 & $4.6 \%$ \\
\hline Total & 18 & 15 & 5 & 78 & 1 & 117 & \\
\hline Percent & $15.4 \%$ & $12.8 \%$ & $4.3 \%$ & $66.7 \%$ & $0.9 \%$ & & \\
\hline
\end{tabular}

MAB M. chelonae ss. abscessus and M. chelonae complex, MAV M. avium, MKA M. kansasii, MIN M. intracellulare, MGO M. gordonae. DM diabetes mellitus, COPD chronic obstructive pulmonary disease, AS Ankylosing 
Table 4 Comparison analysis of comorbidities and clinical features between patients with NTM disease and NTM colonization

\begin{tabular}{lll}
\hline & $\begin{array}{l}\text { Confirmed case } \\
(n=87)\end{array}$ & $\begin{array}{l}\text { Not meet ATS/IDSA criteria } \\
(n=67)\end{array}$ \\
\hline Male & $51(58.6 \%)$ & $40(59.7 \%)$ \\
Female & $36(41.4 \%)$ & $27(40.3 \%)$ \\
Age (median) & $60(20-82)$ & $66(19-87)$ \\
Radiographic characteristic & $25(37.3 \%)$ \\
Cavity & $33(37.9 \%)$ & $28(41.8 \%)$ \\
Nodule & $33(37.9 \%)$ & $26(38.8 \%)$ \\
Bronchiectasis & $34(39.1 \%)$ & $30(44.8 \%)^{*}$ \\
Comorbidities & & $0^{*}$ \\
$\quad \begin{array}{l}\text { Previous TB } \\
\text { HIV positive }\end{array}$ & $56(64.4 \%)^{*}$ & \\
Clinical features & $17(19.5 \%)^{*}$ & $50(74.6 \%)$ \\
Cough and & $68(78.1 \%)$ & $16(23.9 \%)$ \\
Expectoration & & $6(9.0 \%)$ \\
Hemoptysis & $17(19.5 \%)$ & $3(4.5 \%)$ \\
Chest Congestion & $8(9.2 \%)$ & $9(13.4 \%)^{*}$ \\
Shortness of breath & $9(10.3 \%)$ & $24(27.6 \%)^{*}$ \\
Fever & * &
\end{tabular}

*indicates $p<0.05$ for comparison between columns by Chi-square test

and chest congestion. These symptoms were very similar to tuberculosis [30, 31]. Even though the radiographic characteristics of NTM infection often overlap with MTB [32], we did find something special. The main radiographic presentation of NTM pulmonary disease was bronchiectasis (39.1\%), followed by pulmonary cavity (37.9\%) and nodules (37.9\%). NTM lung disease and bronchiectasis are inextricably linked [33]. NTM infection is very common in bronchiectasis patients [34]. So far, it is still not clear whether NTM are a cause or consequence of bronchiectasis [33]. For pulmonary cavity, the significant point is that multiple and thin-walled cavities were predominant in our NTM disease patients. As NTM frequently co-infects with MTB, it is hard to distinguish NTM and MTB from the cavity feature. However, the multiple cavities indicated the lung structure was severely destroyed and could be predisposed to NTM infection.

As NTM are environmental bacteria, a substantial number of patients have no known risk factors. However, some underlying host risk factors for NTM infection have been extensively studied, like allogeneic hematopoietic cell transplantation (AHCT) [35-37]. Remarkably, the patients with predisposed lung disease are more susceptible to NTM infection [30, 38]. Through review of medical records, we found that 64\% NTM patients suffered from previous MTB, and 34 (39.1\%) had bronchiectasis. In our study, though 34 patients had bronchiectasis on radiology, only 17 patients displayed clinical bronchiectasis symptoms. We also observed COPD in 6 (6.9\%) NTM disease patients. Besides heart disease and stroke, COPD is within the top three causes of human death in the world, which frequently appears together with TB [39]. It's reported that $M$. intracellulare lung disease was more likely to be observed among patients with COPD comorbidity [23]. Meanwhile, NTM infection are more often observed in HIV patients [5] and cystic fibrosis (CF) patients [40]. $M$. avium complex is the major causative agent of NTM disease in AIDS or CF patients [5, 40]. Consistently, in our study, $M$. avium was also the most frequently isolated NTM in HIV positive patients.

Interestingly, we did not find any remarkable difference between patients with NTM disease and NTM colonization, with regards to demographic feature, radiographic characteristic and clinical manifestation except fever. This was not surprising, because the 67 patients with NTM colonization were TB patients at the same time, 37 with primary $\mathrm{TB}$ and 30 with secondary TB. It is difficult to differentiate the clinical symptoms between NTM diseases and TB. Meanwhile, it's worth noting that 17 of 87 NTM disease patients was HIV-positive. HIV infection usually impairs host immune system and increases opportunistic infection, which might explain why NTM disease patients presented with more fever in our study. Actually, weak immune system and underlying lung disease are included in the risk factor list for NTM disease [39]. Since they had variable lung destruction, those patients with NTM colonization might meet the NTM lung disease criteria in the future and therefore should have closer follow-up.

Taken together, the significance of NTM lung disease has been brought to the forefront. Investigation and analysis of local prevalence of NTM would be worthwhile. Multicentric study is urgently needed to research the epidemiology and other risk factors responsible for NTM infection.

\section{Conclusions}

This retrospective study showed that the most common pathogenic NTM species in Nanjing district was $M$. intracellulare. NTM pulmonary disease was associated with age, instead of gender. The most frequent radiographic presentation of NTM lung disease was bronchiectasis. Previous TB and HIV infection immensely enhanced risk of NTM disease.

\section{Abbreviations}

AHCT: Allogeneic hematopoietic cell transplantation; AIDS: Acquired Immune Deficiency Syndrome; AS: Ankylosing; ATS/IDSA: American Thoracic Society and Infectious Disease Society of America; CDC: Centers for Disease Control; CF: Cystic fibrosis; COPD: Chronic obstructive pulmonary disease;

DM: Diabetes mellitus; HIV: Human immunodeficiency virus;

MTB: Mycobacterium tuberculosis; NTM: nontuberculous mycobacteria;

TB: Tuberculosis; WHO: World health organization 


\section{Acknowledgements}

Not applicable.

\section{Consent to publication}

Not applicable.

\section{Authors' contributions}

$\mathrm{CMH}$ and LLH conducted the literature review and wrote the draft. MC conducted the statistical analysis. WXW and XDS collected the clinical data. WC conceived the study and revised the manuscript. All authors have read and approved the manuscript.

\section{Funding}

This work was supported by Nanjing medical science and technology development foundation (Grant No. YKK17172) and social development project of key research and development plan of Jiangsu province (Grant No. BE2018606). The former was used to support data query and the latter paid the article-processing fee.

\section{Availability of data and materials}

Data relating to this study are contained and presented in this document. Other materials are available from the corresponding author on reasonable request.

\section{Ethics approval and consent to participate}

Ethical approval was obtained from Institutional Review Board of the Second Hospital of Nanjing for this study (2018-LS-ky017). Receiving informed consent from study participants was exempted because we used medical records reviewing without personal information. Request of original data needs permission of Department of Science and Education in the Second Hospital of Nanjing.

\section{Competing interests}

The authors declare that they have no competing interests.

\section{Author details}

'Department of Tuberculosis, the second hospital of Nanjing, Nanjing University of Chinese Medicine, Nanjing 210003, China. ${ }^{2}$ Department of Science and Education, the second hospital of Nanjing, Nanjing University of Chinese Medicine, Nanjing 210003, China. ${ }^{3}$ Clinical Research Center, the second hospital of Nanjing, Nanjing University of Chinese Medicine, Zhongfu Road 1, Gulou District, Nanjing 210003, Jiangsu, China. ${ }^{4}$ Clinical Laboratory, the second hospital of Nanjing, Nanjing University of Chinese Medicine, Nanjing 210003, China.

\section{Received: 28 December 2018 Accepted: 26 August 2019} Published online: 02 September 2019

\section{References}

1. Primm TP, Falkinham JO. Nontuberculous mycobacteria. In: Quah SR, editor. International Encyclopedia of Public Health (Second Edition). Oxford: Academic Press; 2017. p. 257-63.

2. Falkinham JO. Nontuberculous mycobacteria: community and nosocomial waterborne opportunistic pathogens. Clin Microbiol Newsl. 2016;38(1):1-7.

3. Jeon D. Infection source and epidemiology of nontuberculous mycobacterial lung disease. Tuberc Respir Dis. 2018:81.

4. Kim S-Y, Han SA, Kim DH, Koh W-J. Nontuberculous mycobacterial lung disease: ecology, microbiology, pathogenesis, and antibiotic resistance mechanisms. Precis Future Med. 2017:1(3):99-114.

5. Henkle E, Winthrop KL. Nontuberculous mycobacteria infections in immunosuppressed hosts. Clin Chest Med. 2015;36(1):91-9.

6. Willemse SH, Oomens MAEM, De Lange J, Karssemakers LHE. Diagnosing nontuberculous mycobacterial cervicofacial lymphadenitis in children: a systematic review. Int J Pediatr Otorhinolaryngol. 2018;112:48-54.

7. Ebrahimi G, Farshidpour M, Allen MB, Mirsaeidi M, Falkinham lii JO Nontuberculous mycobacterial disease in North America. Int J Mycobacteriol. 2015;4:154-5.

8. Martínez González S, Cano Cortés A, Sota Yoldi LA, García García JM, Alba Álvarez LM, Palacios Gutiérrez JJ. Non-tuberculous mycobacteria. An emerging threat? Archivos de Bronconeumología (English Edition). 2017; 53(10):554-60
9. Donohue MJ. Increasing nontuberculous mycobacteria reporting rates and species diversity identified in clinical laboratory reports. BMC Infect Dis. 2018;18(1):163.

10. Abubakar I, Gupta RK, Rangaka MX, Lipman M. Update in tuberculosis and nontuberculous mycobacteria 2017. Am J Respir Crit Care Med. 2018; 197(10):1248-53.

11. Shahraki AH, Heidarieh P, Bostanabad SZ, Khosravi AD, Hashemzadeh M, Khandan S, Biranvand M, Schraufnagel DE, Mirsaeidi M. "Multidrug-resistant tuberculosis" may be nontuberculous mycobacteria. Eur J Intern Med. 2015; 26(4):279-84.

12. Philley JV, Griffith DE. Medical Management of Pulmonary Nontuberculous Mycobacterial Disease. Thorac Surg Clin. 2019;29(1):65-76.

13. Zweijpfenning SMH, Ingen JV, Hoefsloot W. Geographic distribution of nontuberculous mycobacteria isolated from clinical specimens: a systematic review. Semin Respir Crit Care Med. 2018;39(3):336-42.

14. Spaulding AB, Lai YL, Zelazny AM, Olivier KN, Kadri SS, Prevots DR, Adjemian J. Geographic distribution of nontuberculous mycobacterial species identified among clinical isolates in the United States, 2009-2013. Annals American Thoracic Soc. 2017;14(11):1655-61.

15. Nasiri MJ, Dabiri H, Fooladi AAl, Amini S, Hamzehloo G, Feizabadi MM. High rates of nontuberculous mycobacteria isolation from patients with presumptive tuberculosis in Iran. New Microbes New Infect. 2018;21:12-7.

16. Koh W-J, Kwon OJ, Jeon K, Kim TS, Lee KS, Park YK, Bai GH. Clinical significance of nontuberculous mycobacteria isolated from respiratory specimens in Korea. Chest. 2006;129(2):341-8.

17. Chono S, Tanino T, Seki T, Morimoto K. Efficient drug targeting to rat alveolar macrophages by pulmonary administration of ciprofloxacin incorporated into mannosylated liposomes for treatment of respiratory intracellular parasitic infections. J Control Release. 2008;127(1):50-8.

18. Li YM, Tong XL, Xu HT, Ju Y, Cai M, Wang C. Prevalence and antimicrobial susceptibility of Mycobacterium abscessus in a general hospital, China. Biomed Environ Sci. 2016;29(2):85-90

19. Bi S, K-j X, Ji Z-k, B-w Z, J-f S. Sentinel site surveillance of nontuberculous mycobacteria pulmonary diseases in Zhejiang, China, 2011-2013. Braz J Infect Dis. 2015;19(6):670-1.

20. Li G, Pang H, Guo Q, Huang M, Tan Y, Li C, Wei J, Xia Y, Jiang Y, Zhao X, et al. Antimicrobial susceptibility and MIC distribution of 41 drugs against clinical isolates from China and reference strains of nontuberculous mycobacteria. Int J Antimicrob Agents. 2017;49(3):364-74.

21. Cheng G, Xu D, Wang J, Liu C, Zhou Y, Cui Y, Liu H, Wan K, Zhou X. Isolation and identification of multiple drug resistant nontuberculous mycobacteria from organs of cattle produced typical granuloma lesions. Microb Pathog. 2017;107:313-6.

22. Zheng HW, Pang $Y$, He GX, Song YY, Zhao YL. Comparing the genotype and drug susceptibilities between Mycobacterium avium and Mycobacterium intracellulare in China. Biomed Environ Sci. 2017;30(7):517-25.

23. Zhang Z, Pang Y, Wang Y, Cohen C, Zhao Y, Liu C. Differences in risk factors and drug susceptibility between Mycobacterium avium and Mycobacterium intracellulare lung diseases in China. Int J Antimicrob Agents. 2015;45(5): 491-5.

24. Gitteh E, Kweku Otu J, Jobarteh T, Mendy F, Faal-Jawara IT, Ofori-Anyinam $\mathrm{NB}$, Ayorinde A, Secka O, Gehre F. Evaluation of sodium hydroxide-N-acetyll-cysteine and $0.7 \%$ chlorhexidine decontamination methods for recovering Mycobacterium tuberculosis from sputum samples: a comparative analysis (the Gambia experience). Int J Mycobacteriol. 2016;5:S167-8.

25. Chinese Antituberculosis Association. Rules for laboratory examination of tuberculosis. Beijing: People's Medical Publishing House; 2015.

26. The application research of PCR-reverse dot blot hybridization assay for rapid identification of Mycobacterium species in BALF. Exp Lab Med. 2016; 34(4):3.

27. Wang H-Y, Kim H, Kim S, Bang H, Kim D-K, Lee H. Evaluation of PCR-reverse blot hybridization assay for the differentiation and identification of Mycobacterium species in liquid cultures. J Appl Microbiol. 2015;1 18(1):142-51.

28. Griffith DE, Aksamit T, Brown-Elliott BA, Catanzaro A, Daley C, Gordin F, Holland SM, Horsburgh R, Huitt G, lademarco MF, et al. An official ATS/IDSA statement: diagnosis, treatment, and prevention of nontuberculous mycobacterial diseases. Am J Respir Crit Care Med. 2007;175(4).

29. Chan ED, Iseman MD. Slender, older women appear to be more susceptible to nontuberculous mycobacterial lung disease. Gend Med. 2010;7(1):5-18.

30. Weinberger SE, Cockrill BA. Mandel J: 24 - tuberculosis and nontuberculous mycobacteria. In: Weinberger SE, Cockrill BA, Mandel J, editors. Principles of 
Pulmonary Medicine (Seventh Edition). Philadelphia: Content Repository Only! 2019. p. 314-24.

31. Duan H, Han X, Wang Q, Wang J, Wang J, Chu N, Huang H: Clinical significance of nontuberculous mycobacteria isolated from respiratory specimens in a Chinese tuberculosis tertiary care center. Sci Rep 2016, 6: 36299-36299

32. Gopinath K, Singh S. Non-tuberculous mycobacteria in TB-endemic countries: are we neglecting the danger? PLoS Negl Trop Dis. 2010;4(4):e615.

33. Griffith DE, Aksamit TR. Bronchiectasis and nontuberculous mycobacterial disease. Clin Chest Med. 2012;33(2):283-95.

34. Mirsaeidi M, Hadid W, Ericsoussi B, Rodgers D, Sadikot RT. Non-tuberculous mycobacterial disease is common in patients with non-cystic fibrosis bronchiectasis. Int J Infect Dis. 2013;17(11):e1000-4.

35. Chan ED, Iseman MD. Underlying host risk factors for nontuberculous mycobacterial lung disease. Semin Respir Crit Care Med. 2013;34(1):110-23.

36. Beswick JM, Shin E, Uhm J, Michelis FV, Viswabandya A, Lipton JH, Messner HA, Marras T, Kim DDH: Incidence and risk factors for nontuberculous mycobacteria infection after allogeneic hematopoietic cell transplantation. Blood 2015, 126(23):1917-1917.

37. Shteinberg M, Stein N, Adir Y, Ken- Dror S, Shitrit D, Bendayan D, Fuks L, Saliba W. Prevalence, risk factors and prognosis of non tuberculous mycobacteria infection among people with bronchiectasis: a population survey. Eur Respir J. 2018:1702469.

38. Marras TK, Campitelli MA, Kwong JC, Lu H, Brode SK, Marchand-Austin A Gershon AS, Jamieson FB. Risk of nontuberculous mycobacterial pulmonary disease with obstructive lung disease. Eur Respir J. 2016;48(3):928-31.

39. O'Toole RF, Shukla SD, Walters EH. TB meets COPD: an emerging global comorbidity in human lung disease. Tuberculosis. 2015;95(6):659-63.

40. Martiniano SL, Nick JA, Daley CL. Nontuberculous mycobacterial infections in cystic fibrosis. Thorac Surg Clin. 2019;29(1):95-108.

\section{Publisher's Note}

Springer Nature remains neutral with regard to jurisdictional claims in published maps and institutional affiliations.

Ready to submit your research? Choose BMC and benefit from:

- fast, convenient online submission

- thorough peer review by experienced researchers in your field

- rapid publication on acceptance

- support for research data, including large and complex data types

- gold Open Access which fosters wider collaboration and increased citations

- maximum visibility for your research: over $100 \mathrm{M}$ website views per year

At $\mathrm{BMC}$, research is always in progress.

Learn more biomedcentral.com/submissions 